\title{
Can Prostate-Specific Antigen Density Be an Index to Distinguish Patients Who Can Omit Repeat Prostate Biopsy in Patients with Negative Magnetic Resonance Imaging?
}

\author{
Jiwoong Yu (D) \\ Youngjun Boo \\ Minyong Kang \\ Hyun Hwan Sung (D) \\ Byong Chang Jeong \\ Seongil Seo \\ Seong soo Jeon \\ Hyunmoo Lee \\ Hwang Gyun Jeon
}

Department of Urology, Samsung Medical Center, Sungkyunkwan University School of Medicine, Seoul, Republic of Korea
Correspondence: Hwang Gyun Jeon Department of Urology, Samsung Medical Center, Sungkyunkwan University School of Medicine, 8I Irwon-Ro, Gangnam-Gu, Seoul, 0635I, Republic of Korea

Tel +82-2-2148-9599

Fax +82-2-3410-6992

Email hwanggyun.jeon@samsung.com
Purpose: We evaluated the negative predictive value (NPV) of multiparametric magnetic resonance imaging (mpMRI) in detecting clinically significant prostate cancer (csPCa) according to biopsy setting and prostate-specific antigen density (PSAD) using transperineal template-guided saturation prostate biopsy (TPB) as the reference standard.

Methods: A total of 161 patients with biopsy histories and negative pre-biopsy mpMRI (Prostate Imaging Reporting and Data System version 2 scores of less than 3) participated in the study. TPB was performed on the following indications: "prior negative biopsy" in patients with persistent suspicion of prostate cancer $(\mathrm{n}=91)$ or "confirmatory biopsy" in patients who were candidates for active surveillance $(\mathrm{n}=70)$. The csPCa was defined as a Gleason score of $3+4$ or greater. We calculated the NPV of mpMRI in detecting csPCa according to biopsy history and prostate-specific antigen density (PSAD) and conducted a logistic regression analysis to determine the clinical predicator for the absence of csPCa.

Results: The detection rate of csPCa was $5.5 \%$ in the prior negative biopsy group and $14.3 \%$ in the confirmatory biopsy group $(P=0.057)$. None of the variables in the logistic regression models including PSAD $<0.15 \mathrm{ng} / \mathrm{mL} / \mathrm{cc}$ and prior negative biopsy could predict the absence of csPCa. The NPV of mpMRI in detecting csPCa in patients with a prior negative biopsy worsen from $94.5 \%$ to $93.3 \%$ when combined with PSAD $<0.15 \mathrm{ng} / \mathrm{mL} / \mathrm{cc}$.

Conclusion: Patients with negative mpMRI findings may not omit repeat biopsy even if their prior biopsy histories are negative and PSADs are $<0.15 \mathrm{ng} / \mathrm{mL} / \mathrm{cc}$.

Keywords: early detection of cancer, prostatic neoplasm, prostate-specific antigen density, magnetic resonance imaging, negative predictive value, transperineal prostate biopsy

\section{Introduction}

Performing multiparametric magnetic resonance imaging (mpMRI) in patients with suspicion of prostate cancer (PCa) before prostate biopsy improves detection rates of clinically significant $\mathrm{PCa}(\mathrm{csPCa})$ by $2 \%-12 \%$ and reduces overdiagnosis of clinically non-significant PCa (cnsPCa) by $11 \%-14 \% .^{1-3}$ In repeat biopsy settings, guidelines strongly suggested performing the mpMRI prior to repeat biopsy to identify a target lesion. ${ }^{4,5}$ It is currently recommended to perform a systematic biopsy when mpMRI is negative in a patient with prior negative biopsy. However, the possibility of distinguishing patients that can safely avoid biopsy by risk stratification combining mpMRI findings, clinical data, and other biomarkers has been proposed at the same time. ${ }^{6}$ 
In this context, several studies have combined biopsy histories and other biomarkers to improve the negative predictive values (NPVs) of mpMRI in repeat biopsy setting (Table 1). Falagario et al analyzed different biopsy strategies based on the combined use of prostate-specific antigen density (PSAD) and mpMRI and suggested the individualized risk of missing $\mathrm{PCa}$ and $\mathrm{csPCa}^{7}$ Recent study from Oishi et al investigated the NPVs of mpMRI in repeat biopsy setting using transrectal ultrasound (TRUS)-guided biopsy as the reference standard. They reported that the NPV of mpMRI were improved from $82 \%$ to $100 \%$, when combined with negative biopsy history and PSAD $<0.15 \mathrm{ng} / \mathrm{mL} / \mathrm{cc}^{8}$

Here, we attempted to validate the NPV of mpMRI in a repeat biopsy setting using a median of 36 core transperineal template-guided saturation prostate biopsy (TPB) as the reference standard. In particular, the csPCa detection rate and the NPV of mpMRI were investigated in combination with prior biopsy status and PSAD levels.

\section{Patients and Methods Study Population}

This study was approved by the Institutional Review Board of Samsung Medical Center (approval number: 2018-05-192) and performed in accordance with the principles of the Declaration of Helsinki. We retrospectively reviewed the clinical data of patients who underwent TPB in repeat biopsy setting between May 2017 and December 2020. Patients who underwent mpMRI at our institution and had negative mpMRI reports, defined as a Prostate Imaging Reporting and Data System version 2 (PI-RADS v2) score of less than 3, were included in this study. While those who had undergone any prior surgical or medical treatment for benign prostatic hyperplasia or previously diagnosed $\mathrm{PCa}$ were excluded.

TPB was performed if clinically indicated by 1) a persistent suspicion of $\mathrm{PCa}$ with rising PSA despite a "prior negative biopsy" or 2) a "confirmatory biopsy" in patients with prior cnsPCa who are candidates for active surveillance (AS). Based on biopsy histology, csPCa was defined as a Gleason Score (GS) of $3+4$ or greater, and cnsPCa was defined as a GS of 6.

\section{MRI Protocol and Interpretation}

T1-weighted, T2-weighted, and diffusion-weighted imaging (apparent diffusion coefficient maps and long-b scans) and dynamic gadolinium contrast-enhanced imaging were performed using a 3-tesla MRI instrument
(Intera Achieva TX; Philips Healthcare, Best, the Netherlands) with a phased-array body coil.

All mpMRI findings were scored using the PI-RADS v2 by two dedicated uro-radiologists with more than 10 years of experience in prostate MRI interpretation. The prostate volume was calculated using the ellipsoid formula based on MRI measurements.

\section{Transperineal Template-Guided Saturation Prostate Biopsy Protocol}

The patients were given a cleaning enema and antibiotics before biopsy. All biopsies were performed in an extended lithotomy position under general anesthesia. After a digital rectal exam, biopsy was performed via a standard 5-mminterval brachytherapy grid fixed on a stepper from the medial to the lateral plane in the anterior, middle, and posterior portions of the prostate gland under the guidance of a biplanar TRUS 8848 probe (BK Medical, Herlev, Denmark). All patients underwent 24 or 36 systematic saturation biopsies depending on prostate size relative to $30 \mathrm{cc}$ according to the Ginsburg protocol. ${ }^{9}$ No MRIdirected biopsies were performed in cases with lesions with PI-RADS scores less than 3.

\section{Statistical Analysis}

The Mann-Whitney $U$-test was used for continuous variables, and the chi-square or Fisher's exact test was used for categorical variables. The NPVs of mpMRI in detecting csPCa were calculated according to PSAD category and biopsy history. In addition, we assessed the NPV of MRI in combination with prior negative biopsy history and PSAD cutoff of $0.15 \mathrm{ng} / \mathrm{mL} / \mathrm{cc}$, which had the lowest Youden index. Univariate and multivariable logistic regression analyses were performed to assess the predictors of absent csPCa with clinically relevant parameters including previous biopsy status and PSAD level. Statistical analyses were conducted using the Statistical Package for the Social Sciences version 21.0 (IBM Corp., Armonk, NY, USA). All p-values were two-tailed, and a p-value less than 0.05 was considered statistically significant.

\section{Results}

A total of 452 consecutive men underwent TPB in repeat biopsy setting at our institution between May 2017 and December 2020, and 171 had negative results in prebiopsy mpMRIs. Of the 171 patients with negative mpMRIs, we excluded 6 patients who underwent treatment for benign 


\begin{tabular}{|c|c|c|c|c|c|c|c|}
\hline 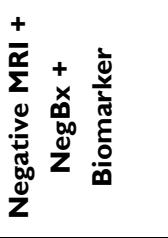 & & ळे & & & ○े & よ̊ & 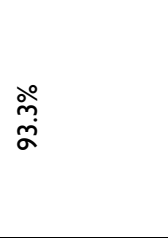 \\
\hline 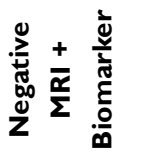 & 응 & ळ̊ & ○े & ○े & ঃ̊ & ஓু & $\begin{array}{l}\text { ํํํ } \\
\text { ळ. }\end{array}$ \\
\hline 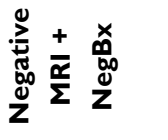 & & 命 & & & ふั & 引ั & 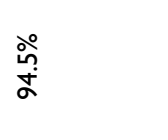 \\
\hline$\frac{\partial}{z} \div \overline{\frac{\alpha}{\Sigma}}$ & ○ें & よ̊ & ஓे & うั & ळે & ஓㅇ & $\begin{array}{l}\stackrel{2}{\hat{2}} \\
\text { 今े }\end{array}$ \\
\hline 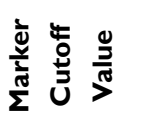 & $\frac{n}{0}$ & $\frac{n}{0}$ & $\stackrel{+}{0}$ & $\stackrel{\stackrel{n}{m}}{ }$ & $\frac{n}{0}$ & $\frac{n}{0}$ & $\frac{n}{0}$ \\
\hline 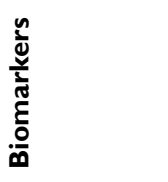 & 号 & 号 & $\stackrel{\varrho}{\frac{I}{1}}$ & 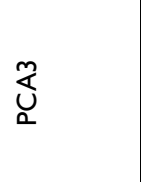 & 号 & 号 & 它 \\
\hline 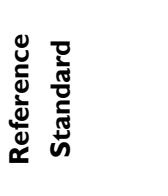 & 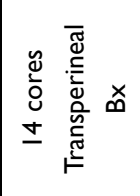 & 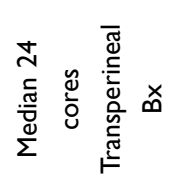 & 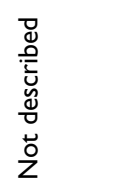 & 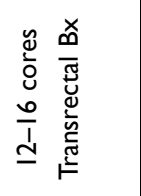 & 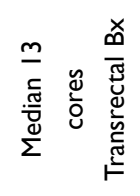 & 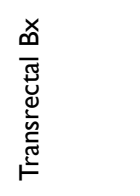 & 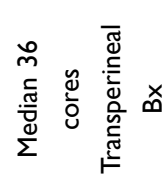 \\
\hline 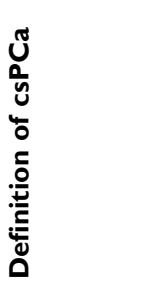 & 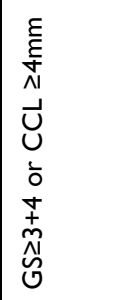 & $\begin{array}{l}+ \\
+ \\
\tilde{N} \\
\stackrel{N}{\circlearrowleft}\end{array}$ & 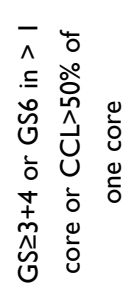 & 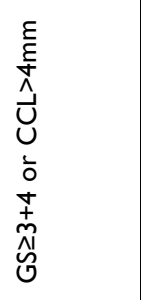 & 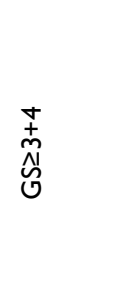 & $\begin{array}{l}+ \\
+ \\
\stackrel{N}{N} \\
\stackrel{N}{\circlearrowleft}\end{array}$ & 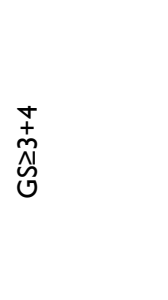 \\
\hline 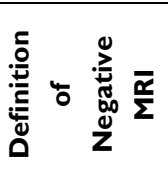 & 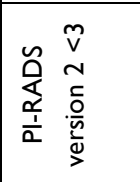 & 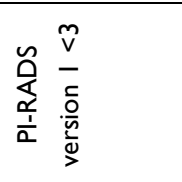 & 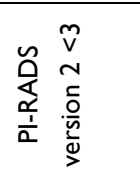 & 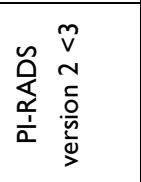 & 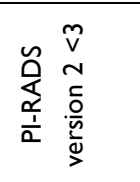 & 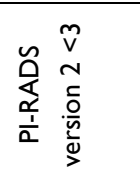 & 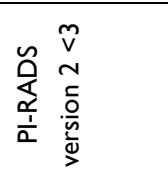 \\
\hline $\begin{array}{l}\frac{1}{2} \\
\frac{0}{5} \\
\frac{0}{2} \\
\frac{0}{0}\end{array}$ & $\begin{array}{l}\hat{I} \\
\\
z \\
x \\
0\end{array}$ & 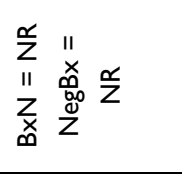 & 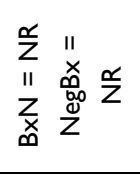 & 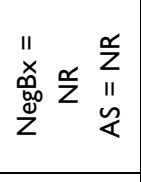 & 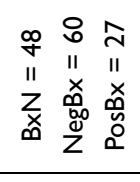 & 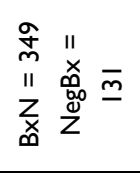 & 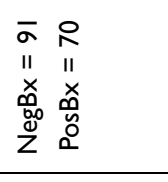 \\
\hline 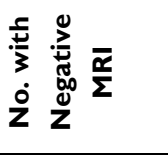 & 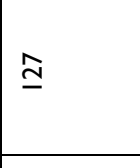 & $\underset{m}{\stackrel{*}{*}}$ & 요 & $\stackrel{\infty}{\sim}$ & $\stackrel{\stackrel{n}{m}}{\underline{m}}$ & $\stackrel{\circ}{\stackrel{\circ}{\sigma}}$ & $\underline{\Xi}$ \\
\hline 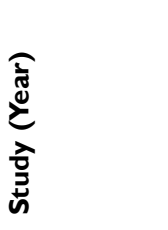 & 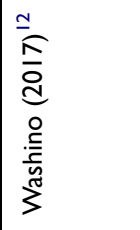 & 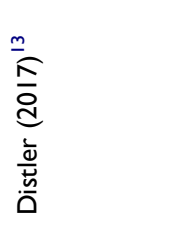 & 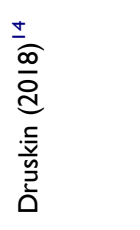 & 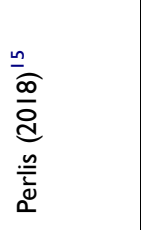 & $\begin{array}{l}\frac{\infty}{\sigma} \\
\frac{a}{2} \\
\frac{\bar{c}}{\bar{m}} \\
\overline{0}\end{array}$ & 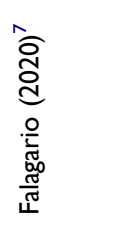 & 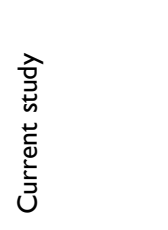 \\
\hline
\end{tabular}


Table 2 Demographics of Patients with Negative MRIs Who Underwent Transperineal Template-Guided Saturation Biopsies

\begin{tabular}{|c|c|c|c|c|}
\hline & \multirow[b]{2}{*}{ Total } & \multicolumn{2}{|c|}{ Biopsy Indication } & \multirow[b]{2}{*}{$\boldsymbol{P}$} \\
\hline & & Prior Negative Biopsy & Confirmatory Biopsy & \\
\hline Number of patients & 161 & 91 & 70 & \\
\hline Median age (IQR) (years) & $62(58-68)$ & $62(58-67)$ & $64(58-69)$ & 0.237 \\
\hline Suspicious DRE, n (\%) & $2(1.2)$ & $2(2.2)$ & $0(0)$ & $0.505^{*}$ \\
\hline Family history of PCa, n (\%) & $9(5.6)$ & $6(6.6)$ & $3(4.3)$ & $0.733^{*}$ \\
\hline Median PSA (IQR) (ng/mL) & $5.6(4.2-8.6)$ & $6.6(4.8-10.0)$ & $4.6(3.6-6.6)$ & $<0.001$ \\
\hline $\begin{array}{l}\text { Median prostate volume (IQR) } \\
\text { (cc) }\end{array}$ & $40.4(31.3-54.8)$ & $43.8(34.8-57.0)$ & $37.7(29.7-50.0)$ & 0.032 \\
\hline Median PSAD (IQR) (ng/mL/cc) & $0.14(0.10-0.20)$ & $0.15(0.11-0.24)$ & $0.13(0.09-0.16)$ & 0.004 \\
\hline$<0.15 \mathrm{ng} / \mathrm{mL} / \mathrm{cc}, \mathrm{n}(\%)$ & $95(59.0)$ & $45(49.5)$ & $50(71.4)$ & 0.005 \\
\hline \multicolumn{5}{|l|}{ Prior TRUS-guided biopsy } \\
\hline Benign prostate tissue & $83(51.6)$ & $83(91.2)$ & $0(0)$ & \\
\hline HGPIN & $5(3.1)$ & $5(5.5)$ & $0(0)$ & \\
\hline ASAP & $3(1.9)$ & $3(3.3)$ & $0(0)$ & \\
\hline $\mathrm{PCa}(\mathrm{GS} \mathrm{3+3)}$ & $70(43.5)$ & $0(0)$ & $70(100)$ & \\
\hline
\end{tabular}

Notes: *Fisher's exact test.

Abbreviations: MRI, magnetic resonance imaging; IQR, interquartile range; DRE, digital rectal examination; PSA, prostate-specific antigen; PSAD, PSA density; TRUS, transrectal ultrasound; HGPIN, high-grade prostatic intraepithelial neoplasia; ASAP, atypical small acinar proliferation; PCa, prostate cancer; GS, Gleason score.

prostatic hyperplasia and 4 patients who received androgen deprivation therapy for PCa diagnosed in a prior biopsy. Therefore, 161 patients met the inclusion criteria for this study. Among this cohort, 91 (56.5\%) patients were indicated for TPB by a persistent suspicion of PCa with rising PSA despite a negative result on prior TRUSguided biopsy, and 70 (43.5\%) patients were indicated for a confirmatory biopsy for the previously detected cnsPCa by TRUS-guided biopsy.

The baseline demographics and characteristics of the patients with negative mpMRIs are summarized in Table 2. Patients in the prior negative biopsy group had significantly higher PSA levels and larger prostate volumes than those in the confirmatory group $(P<0.001$ and $P<0.032$, respectively). PSAD levels were higher in the prior negative biopsy group, and the proportion of patients with $\mathrm{PSAD}<0.15 \mathrm{ng} / \mathrm{mL} / \mathrm{cc}$ was higher in the confirmatory group $(P=0.004$ and $P=0.005$, respectively). Of the 91 patients with prior negative biopsies, 5 $(5.5 \%)$ had previously been diagnosed with high-grade prostatic intraepithelial neoplasia, and 3 (3.3\%) were diagnosed with atypical small acinar proliferation.

The TPB results are presented in Table 3. According to the TPB protocol of our institution, the median number of biopsy cores (interquartile range) was 36 (24-36) in both groups. The detection rate of any $\mathrm{PCa}$ was $23.1 \%$ in the prior negative biopsy group and $58.6 \%$ in the confirmatory biopsy group, including 8 patients with GS $7(3+4) \mathrm{PCa}$ and 2 patients with GS $8(4+4)$ PCa. There was a significant difference in the detection rate of any $\mathrm{PCa}$ between the two groups $(P<0.001)$. However, the detection rate of csPCa was $5.5 \%$ in the prior negative biopsy group and $14.3 \%$ in the confirmatory biopsy group, and there was no statistical difference between the groups $(P=$ $0.057)$.

The NPV of mpMRI in detecting csPCa was $90.7 \%$, $94.5 \%$, and $85.7 \%$ in the overall study population, previous negative biopsy group, and confirmatory biopsy group, respectively (Table 4). In the previous negative biopsy group, the NPV of mpMRI was $100 \%$ in PSAD categories of $0.15-0.20 \mathrm{ng} / \mathrm{mL} / \mathrm{cc}$ and PSAD $<0.10 \mathrm{ng} /$ $\mathrm{mL} / \mathrm{cc}$. However, the PSAD category of $0.10-0.15 \mathrm{ng} /$ $\mathrm{mL} / \mathrm{cc}$ had the lowest NPV at $89.7 \%$. When combined with PSAD $<0.15 \mathrm{ng} / \mathrm{mL} / \mathrm{cc}$, it was found to be lower at $89.5 \%$ (Figure 1). In addition, in the prior negative biopsy group, the NPV of mpMRI was $94.5 \%$ and it was found to worsen at $93.3 \%$ when combined with PSAD $<0.15 \mathrm{ng} /$ $\mathrm{mL} / \mathrm{cc}$. 
Table 3 Results of Transperineal Template-Guided Saturation Biopsy in Patients with Negative MRIs

\begin{tabular}{|c|c|c|c|c|}
\hline & \multicolumn{3}{|c|}{ Biopsy Indication } & \multirow[b]{2}{*}{$P$} \\
\hline & Total & Prior Negative Biopsy & Confirmatory Biopsy & \\
\hline Number of patients & 161 & 91 & 70 & \\
\hline $\begin{array}{l}\text { Median number of biopsy cores } \\
\text { (IQR) }\end{array}$ & $36(24-36)$ & $36(24-36)$ & $36(24-36)$ & 0.959 \\
\hline $\begin{array}{l}\text { Median number of positive cores } \\
\text { (IQR) }\end{array}$ & $0(0-1)$ & $0(0-0)$ & I (0-4) & $<0.001$ \\
\hline \multicolumn{5}{|l|}{ Results of TPB, $n(\%)$} \\
\hline Benign prostate tissue & $93(57.8)$ & $65(71.4)$ & $28(40.0)$ & \\
\hline HGPIN & $2(1.2)$ & $\mathrm{I}(\mathrm{I} . \mathrm{I})$ & I (I.4) & \\
\hline ASAP & $4(2.5)$ & $4(4.4)$ & $0(0)$ & \\
\hline PCa (GS 3+3) & $47(29.2)$ & $16(17.6)$ & $31(44.3)$ & \\
\hline $\mathrm{PCa}(\mathrm{GS} \mathrm{3+4)}$ & II (6.8) & $3(3.3)$ & $8(11.4)$ & \\
\hline PCa (GS 4+3) & $2(1.2)$ & $2(2.2)$ & $0(0)$ & \\
\hline PCa (GS 4+4) & $2(1.2)$ & $0(0)$ & $2(2.9)$ & \\
\hline $\begin{array}{l}\text { Median maximal percent of } \\
\text { cancer in core (IQR) }\end{array}$ & $10(5-30)$ & $10(5-30)$ & $15(10-40)$ & 0.148 \\
\hline Detection of any PCa, $n(\%)$ & $62(38.5)$ & $21(23.1)$ & $4 \mid(58.6)$ & $<0.001$ \\
\hline Detection of csPCa, n (\%) & $15(9.3)$ & $5(5.5)$ & $10(14.3)$ & 0.057 \\
\hline
\end{tabular}

Abbreviations: MRI, magnetic resonance imaging; IQR, interquartile range; TPB, transperineal template-guided saturation biopsy; HGPIN, high-grade prostatic intraepithelial neoplasia; ASAP, atypical small acinar proliferation; GS, Gleason score; PCa, prostate cancer; csPCa, clinically significant prostate cancer.

Univariate and multivariate logistic regression models were established for the analysis of association with the absence of any $\mathrm{PCa}$ or csPCa based on age, family history of PCa, PSA level, prostate volume, PSAD $<0.15 \mathrm{ng} / \mathrm{mL} / \mathrm{cc}$, and the indication of TPB (Table 5). In the univariate model, higher PSA level (OR 1.089, 95\% CI 1.009-1.177, $P=$ 0.029 ) and prior negative biopsy group (OR 4.197, 95\% CI 2.073-8.496, $P<0.0001)$ were predictive of the absence of any PCa. The multivariate model revealed that only the prior negative biopsy group was predictive of the absence of any PCa (OR 4.197, 95\% CI 2.073-8.496; $P<0.0001$ ). In contrast, none of the variables were predictive of the absence of csPCa on TPB in either univariate or multivariate analysis.

\section{Discussion}

In this retrospective study, we examined the any $\mathrm{PCa}$ and csPCa detection rates in patients who had no suspected lesions on mpMRI (PI-RADS <3) and underwent repeat biopsies using TPB. We also calculated the NPV of mpMRI by combining the prior biopsy histories and PSAD levels. To the best of our knowledge, this is the first study to analyze the NPV of mpMRI scored by PIRADS v2 by performing TPB with a median of 36 cores.

Our institution has performed TPB if clinically indicated by a persistent suspicion of PCa with rising PSA despite a previous negative result on TRUS-guided biopsy and as confirmatory biopsy in patient candidates for AS

Table 4 Negative Predictive Value of MRI According to Prostate-Specific Antigen Density Category and Biopsy History

\begin{tabular}{|l|c|c|c|}
\hline \multirow{2}{*}{ PSAD } & Total & \multicolumn{2}{|c|}{ Biopsy Indication } \\
\cline { 2 - 4 } & & Prior Negative Biopsy & Confirmatory Biopsy \\
\hline Total & $90.7 \%(146 / 161)$ & $94.5 \%(86 / 91)$ & $85.7 \%(60 / 70)$ \\
PSAD $\geq 0.20 \mathrm{ng} / \mathrm{mL} / \mathrm{cc}$ & $92.7 \%(38 / 41)$ & $93.5 \%(29 / 31)$ & $90.0 \%(9 / 10)$ \\
$0.15 \mathrm{ng} / \mathrm{mL} / \mathrm{cc} \leq$ PSAD $<0.20 \mathrm{ng} / \mathrm{mL} / \mathrm{cc}$ & $92.0 \%(23 / 25)$ & $100.0 \%(15 / 15)$ & $80.0 \%(8 / 10)$ \\
$0.10 \mathrm{ng} / \mathrm{mL} / \mathrm{cc} \leq \mathrm{PSAD}<0.15 \mathrm{ng} / \mathrm{mL} / \mathrm{cc}$ & $87.9 \%(51 / 58)$ & $89.7 \%(26 / 29)$ & $86.2 \%(25 / 29)$ \\
PSAD $<0.10 \mathrm{ng} / \mathrm{mL} / \mathrm{cc}$ & $91.9 \%(34 / 37)$ & $100 \%(16 / 16)$ & $85.7 \%(18 / 21)$ \\
\hline
\end{tabular}

Abbreviations: MRI, magnetic resonance imaging; PSAD, prostate-specific antigen density. 


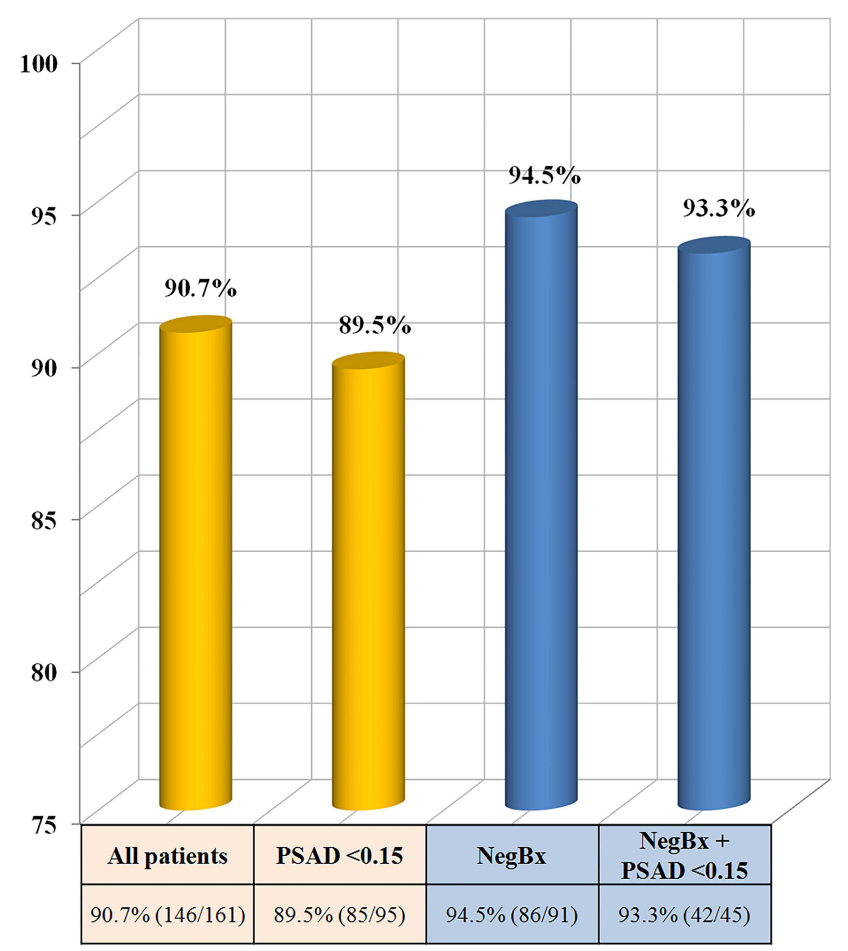

Figure I Negative predictive value of multiparametric magnetic resonance imaging in detecting clinically significant prostate cancer in combination with prior negative biopsy history and PSAD cutoff of $0.15 \mathrm{ng} / \mathrm{mL} / \mathrm{cc}$.

Abbreviations: NegBx, prior negative biopsy; PSAD, prostate-specific antigen density $(\mathrm{ng} / \mathrm{mL} / \mathrm{cc})$.

after a prior detected cnsPCa on TRUS-guided biopsy. In $91(56.5 \%)$ patients with prior negative biopsies and 70 (43.5\%) patients with prior positive biopsies, the histology of a median of 36 cores of TPB using 5-mm sampling was used as the reference standard. The incidence of biopsydetected csPCa was $5.5 \%$ in men with negative mpMRI and prior negative biopsy history, and $14.3 \%$ in men with negative mpMRI and prior positive biopsy history. The NPV of mpMRI in the detection of csPCa in patients with prior negative biopsies worsen from $94.5 \%$ to 93.3\% when combined with PSAD $<0.15 \mathrm{ng} / \mathrm{mL} / \mathrm{cc}$.

The multi-institutional Prostate MR Imaging Study (PROMIS) trial evaluated the detection rate of $\mathrm{PCa}$ in 576 patients who had undergone prebiopsy mpMRI with saturation transperineal prostate biopsy as the reference standard. ${ }^{10}$ Of the 158 patients with negative mpMRI in the study, 141 patients reported having no cancer or nonsignificant cancer (GS 6) on transperineal prostate biopsy, and the NPV of mpMRI predicting the absence of GS 7 (3 $+4)$ or greater cancer was $89 \%$ (95\% confidence interval $83 \%-94 \%$ ). PROMIS trial presents the possibility of reducing the proportion of men with unnecessary biopsies. However, limitations of the prebiopsy mpMRI were also reported. Vargas et al confirmed that PI-RADS v2 was limited for the assessment of GS $\geq 4+3$ tumors with a volume of less than $0.5 \mathrm{~mL} .{ }^{11}$ In addition, in situations where approximately $30 \%$ to $40 \%$ of the patients have been reported to have negative results on prebiopsy MRIs, it is not yet clear which patients can safely omit the biopsy.

There have been several reports on improving the NPV of mpMRI in combination with clinical information with negative mpMRI findings (Table 1)..$^{8,12-15}$ In particular, two studies of Perlis et al and Oishi et al evaluated the NPV of mpMRI by adding prior biopsy histories and different biomarkers using TRUS-guided biopsy as the reference standard. Perlis et al investigated patients with a previous negative biopsy and patients on AS and reported that with a negative multiparametric MRI and normal prostate cancer antigen 3 (PCA3) score, no csPCa was detected on repeat TRUS-guided biopsy. ${ }^{15}$ Therefore, they suggested that repeat biopsies may not be needed in patients with dual negative results (negative results from both mpMRI and PCA3 scores). A recent study by Oishi et al reported outcomes of repeat biopsy in 135 patients with negative prebiopsy mpMRIs. ${ }^{8}$ The detection rate of csPCa (GS $3+4$ or greater) was $18 \%$ in men with negative mpMRIs. The NPV of mpMRI was $100 \%$ for patients with prior negative biopsies who had PSAD $<0.15 \mathrm{ng} / \mathrm{mL} / \mathrm{cc}$, and the authors proposed that this subset of patients could avoid repeat biopsy.

However, because the reference standard of the aforementioned two studies was TRUS-guided biopsy, information regarding omitting biopsy might be limited. The TRUS-guided biopsy can be associated with both the underdetection of csPCa and the increased diagnosis of many cnsPCas. ${ }^{10,16,17}$ In addition, in TRUS-guided biopsies, either the size or the grade of PCa may be underestimated due to random sampling error. ${ }^{18}$ Furthermore, the pathological status derived from TRUS-guided biopsies in the repeat biopsy setting can be unreliable in discriminating csPCa from cnsPCa, such that $34 \%$ of the men have been found to have an upgrading of $\mathrm{PCa} .{ }^{19}$ Thus, TRUS-guided biopsy may not be a sufficient modality to determine the NPV of prebiopsy mpMRI. Despite meeting the criteria for combining negative MRI results and other clinical information or biomarkers, patients could still harbor risk of csPCa, and whether biopsies can be omitted in these patients remains unclear. In patients with prior negative biopsy of this present study, PSAD category of $0.10-0.15 \mathrm{ng} / \mathrm{mL} / \mathrm{cc}$ had the lowest NPV. Practically, in 


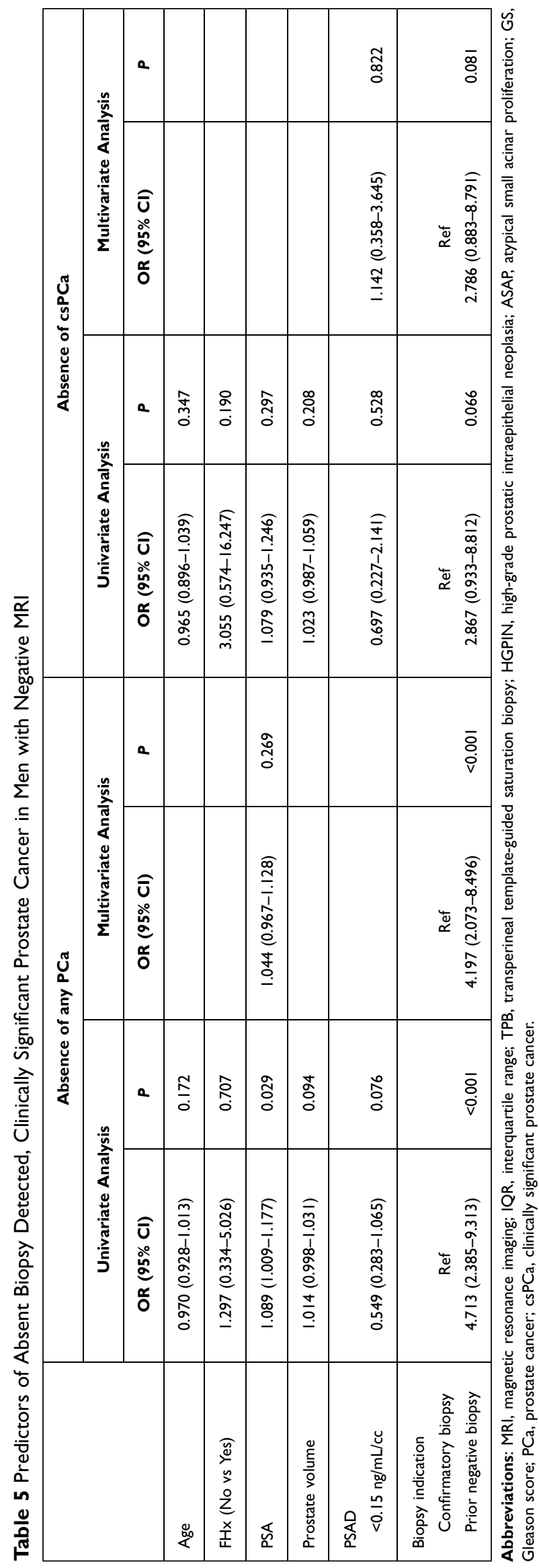

our study, among the five patients diagnosed with csPCa in the prior negative biopsy group, three patients had PSAD $<0.15 \mathrm{ng} / \mathrm{mL} / \mathrm{cc}(0.10,0.11$, and $0.14 \mathrm{ng} / \mathrm{mL} / \mathrm{cc})$. Furthermore, the logistic regression analysis showed none of the variables were predictive of the absence of csPCa. These results suggest that there would be limitations in distinguishing between patients who can safely avoid biopsy with PSAD, even in patients with prior negative biopsy and negative MRI.

In contrast to TRUS-guided biopsy, TPB has very high sensitivity and specificity for csPCa detection, and the results of TPB more accurately reflect the subsequent pathological results of radical prostatectomy than those of TRUS-guided biopsy. ${ }^{20-22}$ The TPB has the advantage of approaching the anterior prostate and the transitional zone and produces a histological map of the entire prostate gland. ${ }^{23-25}$ This biopsy modality not only produces a three-dimensional map of the entire prostate gland but also evaluates small lesions $(<0.5 \mathrm{~mL})$ that mpMRI may not assess. ${ }^{13,26,27}$

There was a previous study of the NPV of mpMRI using transperineal approach. Distler et al combined mpMRI with PSAD to predict the detection rate of csPCa (GS $3+4$ or greater) by performing transperineal systematic biopsy in biopsy-naive or previously negative patients. ${ }^{13}$ They described the NPV of mpMRI for detecting csPCa in patients with negative mpMRI (PI-RADS version 1 score less than 3). Unlike our study, the NPV of mpMRI in patients with prior negative biopsies increased from $83 \%$ to $93 \%$ in combination with PSAD $<0.15 \mathrm{ng} / \mathrm{mL} / \mathrm{cc}$. This might be explained that the NPV of mpMRI was too low due to the low sensitivity of PIRADS version 1; thus, this could be interpreted that the NPV improvement was observed when PSAD was combined. In our study, the NPV of mpMRI in patients with a prior negative biopsy was $94.5 \%$, which was much higher than the result of the study (83\%). Statistically, as the sensitivity of a test increases, the NPV also increases. Regarding the sensitivities of PI-RADS version 1 and PIRADS v2, Tewes et al evaluated the diagnostic accuracy according to PI-RADS version 1 and PI-RADS v2 of two independent readers, and reported sensitivities of reader 1 and reader 2 of $84 \%$ and $74 \%$ for PI-RADS version 1 , respectively, and $100 \%$ and $81 \%$ for PI-RADS v2, respectively. ${ }^{28} \mathrm{~A}$ head-to-head comparison of PI-RADS version 1 and PI-RADS v2 stratified by cancer location reported higher sensitivity of PI-RADS v2 than version 1 (87.5-100\% vs $75 \%)$ in transitional zone cancer, and the 
similar sensitivity of the two versions in peripheral zone cancer (both 100\%). ${ }^{29}$ Furthermore, the relatively low prevalence of $\mathrm{PCa}$ in our Asian cohort compared to that in the Western population could affect higher NPV of mpMRI. ${ }^{30,31}$

Our study also has several limitations. First, due to its nonrandomized retrospective cohort study design, the patient information in our data might be incomplete. Because our institution also performed repeat prostate biopsy with the transrectal approach in some patients who did not want general anesthesia, selection bias that can impact the results could not be eradicated. Second, the cohort size was relatively small. However, TPB under general anesthesia was used to obtain more detailed information on the prostate of the participants. Furthermore, to maintain the uniformity of our study cohort, we excluded patients who had undergone medical or surgical treatment for benign prostatic hyperplasia or PCa. Third, the present study focused primarily on the pathological findings from biopsies, but not radical prostatectomy specimens. Nevertheless, we performed TPB in all patients with the most accurate biopsy modality to reflect the pathologic results of the prostatectomy specimens. ${ }^{21}$ Finally, MRI scans were not specifically reviewed in this study. Nonetheless, highquality mpMRI was performed with an identical instrument for all patients, and two experienced uro-radiologists interpreted and reported the images according to the PI-RADS criteria. $^{5}$

\section{Conclusion}

The NPV of mpMRI in patients with a prior negative biopsy did not improve when PSAD was combined. Patients with negative MRIs, prior negative biopsies and PSAD $<0.15 \mathrm{ng} / \mathrm{mL} / \mathrm{cc}$ may not omit repeat biopsies, as some patients could be diagnosed with csPCa, especially when performing TPB.

\section{Ethical Approval}

This study was approved by the institutional review board at Samsung Medical Center and abided with the Helsinki Declaration. For this type of study, formal consent was not required and data were anonymous.

\section{Acknowledgments}

The authors thank Statistics and Data Center at Samsung Medical Center for their statistical support.

\section{Author Contributions}

All authors made substantial contributions to conception and design, acquisition of data, or analysis and interpretation of data; took part in drafting the article or revising it critically for important intellectual content; agreed to submit to the current journal; gave final approval of the version to be published; and agree to be accountable for all aspects of the work.

\section{Disclosure}

All authors have no direct or indirect conflict of interest with any institution or product.

\section{References}

1. Kasivisvanathan V, Rannikko AS, Borghi M, et al. MRI-targeted or standard biopsy for prostate-cancer diagnosis. $N$ Engl $J$ Med. 2018;378(19):1767-1777. doi:10.1056/NEJMoa1801993

2. van der Leest M, Cornel E, Israel B, et al. Head-to-head Comparison of transrectal ultrasound-guided prostate biopsy versus multiparametric prostate resonance imaging with subsequent magnetic resonance-guided biopsy in biopsy-naive men with elevated prostate-specific antigen: a large prospective multicenter clinical study. Eur Urol. 2019;75(4):570-578. doi:10.1016/j. eururo.2018.11.023

3. Rouviere O, Puech P, Renard-Penna R, et al. Use of prostate systematic and targeted biopsy on the basis of multiparametric MRI in biopsy-naive patients (MRI-FIRST): a prospective, multicentre, paired diagnostic study. Lancet Oncol. 2019;20(1):100-109. doi:10.1016/S1470-2045(18)30569-2

4. Mottet N, Bellmunt J, Bolla M, et al. EAU-ESTRO-SIOG Guidelines on Prostate Cancer. Part 1: screening, Diagnosis, and Local Treatment with Curative Intent. Eur Urol. 2017;71(4):618-629. doi:10.1016/j.eururo.2016.08.003

5. Rosenkrantz AB, Verma S, Choyke P, et al. Prostate Magnetic Resonance Imaging and Magnetic Resonance Imaging Targeted Biopsy in Patients with a Prior Negative Biopsy: a Consensus Statement by AUA and SAR. J Urol. 2016;196(6):1613-1618. doi:10.1016/j.juro.2016.06.079

6. Mottet N, van den Bergh RCN, Briers E, et al. EAU-EANM-ESTROESUR-SIOG Guidelines on Prostate Cancer-2020 Update. Part 1: screening, Diagnosis, and Local Treatment with Curative Intent. Eur Urol. 2021;79(2):243-262.

7. Falagario UG, Jambor I, Lantz A, et al. Combined use of prostate-specific antigen density and magnetic resonance imaging for prostate biopsy decision planning: a retrospective multi-institutional study using the Prostate Magnetic Resonance Imaging Outcome Database (PROMOD). Eur Urol Oncol. 2020. doi:10.1016/j.euo.2020.08.014

8. Oishi M, Shin T, Ohe C, et al. Which patients with negative magnetic resonance imaging can safely avoid biopsy for prostate cancer? J Urol. 2019;201(2):268-276. doi:10.1016/j.juro.2018.08.046

9. Kuru TH, Wadhwa K, Chang RT, et al. Definitions of terms, processes and a minimum dataset for transperineal prostate biopsies: a standardization approach of the Ginsburg Study Group for Enhanced Prostate Diagnostics. BJU Int. 2013;112(5):568-577. doi:10.1111/bju. 12132

10. Ahmed HU, El-Shater Bosaily A, Brown LC, et al. Diagnostic accuracy of multi-parametric MRI and TRUS biopsy in prostate cancer (PROMIS): a paired validating confirmatory study. Lancet. 2017;389(10071):815-822. doi:10.1016/S0140-6736(16)32401-1 
11. Vargas HA, Hotker AM, Goldman DA, et al. Updated prostate imaging reporting and data system (PIRADS v2) recommendations for the detection of clinically significant prostate cancer using multiparametric MRI: critical evaluation using whole-mount pathology as standard of reference. Eur Radiol. 2016;26(6):1606-1612. doi:10.1007/s00330-015-4015-6

12. Washino S, Okochi T, Saito K, et al. Combination of prostate imaging reporting and data system (PI-RADS) score and prostate-specific antigen (PSA) density predicts biopsy outcome in prostate biopsy naive patients. BJU Int. 2017;119(2):225-233.

13. Distler FA, Radtke JP, Bonekamp D, et al. The value of PSA density in cmbination with PI-RADS for the accuracy of prostate cancer prediction. $J$ Urol. 2017;198(3):575-582. doi:10.1016/j. juro.2017.03.130

14. Druskin SC, Tosoian JJ, Young A, et al. Combining Prostate Health Index density, magnetic resonance imaging and prior negative biopsy status to improve the detection of clinically significant prostate cancer. BJU Int. 2018;121(4):619-626. doi:10.1111/bju.14098

15. Perlis N, Al-Kasab T, Ahmad A, et al. Defining a cohort that may not require repeat prostate biopsy based on PCA3 score and magnetic resonance imaging: the dual negative effect. J Urol. 2018;199 (5):1182-1187. doi:10.1016/j.juro.2017.11.074

16. Bangma $\mathrm{CH}$, Roemeling S, Schroder FH. Overdiagnosis and overtreatment of early detected prostate cancer. World J Urol. 2007;25 (1):3-9. doi:10.1007/s00345-007-0145-z

17. Scattoni V, Zlotta A, Montironi R, Schulman C, Rigatti P, Montorsi F. Extended and saturation prostatic biopsy in the diagnosis and characterisation of prostate cancer: a critical analysis of the literature. Eur Urol. 2007;52(5):1309-1322. doi:10.1016/j.eururo.2007.08.006

18. Kulkarni GS, Al-Azab R, Lockwood G, et al. Evidence for a biopsy derived grade artifact among larger prostate glands. J Urol. 2006;175 (2):505-509. doi:10.1016/S0022-5347(05)00236-3

19. Porten SP, Whitson JM, Cowan JE, et al. Changes in prostate cancer grade on serial biopsy in men undergoing active surveillance. J Clin Oncol. 2011;29(20):2795-2800. doi:10.1200/JCO.2010.33.0134

20. Thompson JE, Hayen A, Landau A, et al. Medium-term oncological outcomes for extended vs saturation biopsy and transrectal vs transperineal biopsy in active surveillance for prostate cancer. BJU Int. 2015;115(6):884-891. doi:10.1111/bju.12858

21. Crawford ED, Rove KO, Barqawi AB, et al. Clinical-pathologic correlation between transperineal mapping biopsies of the prostate and three-dimensional reconstruction of prostatectomy specimens. Prostate. 2013;73(7):778-787. doi:10.1002/pros.22622
22. Bott SR, Henderson A, Halls JE, Montgomery BS, Laing R, Langley SE. Extensive transperineal template biopsies of prostate: modified technique and results. Urology. 2006;68(5):1037-1041. doi:10.1016/j.urology.2006.05.033

23. Takenaka A, Hara R, Ishimura $\mathrm{T}$, et al. A prospective randomized comparison of diagnostic efficacy between transperineal and transrectal 12-core prostate biopsy. Prostate Cancer Prostatic Dis. 2008;11(2):134-138. doi:10.1038/sj.pcan.4500985

24. Cerruto MA, Vianello F, D'Elia C, Artibani W, Novella G. Transrectal versus transperineal 14-core prostate biopsy in detection of prostate cancer: a comparative evaluation at the same institution. Arch Ital Urol Androl. 2014;86(4):284-287. doi:10.4081/ aiua.2014.4.284

25. Onik G, Barzell W. Transperineal 3D mapping biopsy of the prostate: an essential tool in selecting patients for focal prostate cancer therapy. Urol Oncol. 2008;26(5):506-510. doi:10.1016/j.urolonc.2008.03.005

26. Lee CU, Sung SH, Jang CT, et al. Cancer location in upgrading and detection after transperineal template-guided mapping biopsy for patients in active surveillance and negative transrectal ultrasonography-guided prostate biopsy. Urol Int. 2019;103 (3):262-269. doi:10.1159/000501527

27. Song W, Kang M, Jeong BC, et al. The clinical utility of transperineal template-guided saturation prostate biopsy for risk stratification after transrectal ultrasound-guided biopsy. Investig Clin Urol. 2019;60 (6):454-462. doi:10.4111/icu.2019.60.6.454

28. Tewes S, Mokov N, Hartung D, et al. Standardized reporting of prostate MRI: comparison of the Prostate Imaging Reporting and Data System (PI-RADS) Version 1 and Version 2. PLoS One. 2016;11(9):e0162879. doi:10.1371/journal.pone.0162879

29. Polanec S, Helbich TH, Bickel H, et al. Head-to-head comparison of PI-RADS v2 and PI-RADS v1. Eur J Radiol. 2016;85(6):1125-1131. doi:10.1016/j.ejrad.2016.03.025

30. Han HH, Park JW, Na JC, Chung BH, Kim CS, Ko WJ. Epidemiology of prostate cancer in South Korea. Prostate Int. 2015;3(3):99-102. doi:10.1016/j.prnil.2015.06.003

31. Lee HY, Kim DK, Doo SW, et al. Time trends for prostate cancer incidence from 2003 to 2013 in South Korea: an age-period-cohort analysis. Cancer Res Treat. 2020;52(1):301-308. doi:10.4143/ crt.2019.194
Cancer Management and Research

\section{Publish your work in this journal}

Cancer Management and Research is an international, peer-reviewed open access journal focusing on cancer research and the optimal use of preventative and integrated treatment interventions to achieve improved outcomes, enhanced survival and quality of life for the cancer patient.
Dovepress

The manuscript management system is completely online and includes a very quick and fair peer-review system, which is all easy to use. Visit http://www.dovepress.com/testimonials.php to read real quotes from published authors. 